\title{
Ontogenetic distribution of Callinectes ornatus (Decapoda, Portunoidea) in southeastern Brazil
}

\section{Distribución ontogenética de Callinectes ornatus (Decapoda, Portunoidea) en el sureste de Brasil}

\author{
Luciana Segura de Andrade ${ }^{1 *}$, Vívian Fransozo², Valter José Cobo ${ }^{3}$, Antônio Leão Castilho1,
} Giovana Bertini ${ }^{4}$, Adilson Fransozo ${ }^{1}$

${ }^{1}$ Departamento de Zoologia, Instituto de Biociências, NEBECC (Crustacean Biology, Ecology and Culture Study Group), Universidade Estadual Paulista, Campus de Botucatu, Distrito de Rubião Junior, s/n, 18618000, Botucatu, SP, Brazil.

2 Departamento de Ciências Naturais, Universidade Estadual do Sudoeste da Bahia, Estrada do Bem Querer, Km 04, 45031-900, Vitória da Conquista, BA, Brazil.

${ }^{3}$ Laboratório de Zoologia, Departamento de Biologia, Universidade de Taubaté. Praça Marcelino Monteiro 63, 12030-010, Taubaté, São Paulo, Brazil.

${ }^{4}$ Universidade Estadual Paulista, Campus Experimental de Registro, Rua Nelson Brihi Badur, 430, 11900-000, Registro, SP, Brazil.

* Corresponding author. E-mail: andradels@ibb.unesp.br

\begin{abstract}
The spatial and seasonal distribution of different demographic groups of the swimming crab Callinectes ornatus was analyzed with respect to its population biology in three bays (Ubatumirim, Ubatuba, and Mar Virado) in southeastern Brazil. In each bay, monthly sampling was performed during two years along six transects, established at 5, 10, 15, and $20 \mathrm{~m}$ depth (parallel to the beach line), in a wave-sheltered area $(7.5 \mathrm{~m}$ depth), and at an exposed site $(10 \mathrm{~m}$ depth). Total abundance of crabs was similar among the bays. The presence of immature individuals, adult females, and breeding females was positively correlated with the bottom and surface temperatures, whereas adult males showed an opposite trend with respect to these factors. Immature and smaller individuals were most abundant along the sheltered and shallower transects. Males were more abundant along the shallower transects, and females at 15 and $20 \mathrm{~m}$. This study revealed that different demographic groups occupy the habitat in different ways, according to local features. In general, southeastern Brazil offers a range of conditions that favor reproduction and consequently the maintenance and growth of the local population of $C$. ornatus, contributing to the relatively high abundance of the species in this coastal area.
\end{abstract}

Key words: coastal monitoring, differential habitat occupation, ontogeny, predatory fishing, swimming crabs.

RESUMEN. Se analizó la distribución espacial y temporal de distintos grupos demográficos del cangrejo nadador Callinectes ornatus en cuanto a su biología poblacional en tres bahías (Ubatumirim, Ubatuba y Mar Virado) en el sureste de Brasil. En cada bahía se realizaron muestreos mensuales durante dos años en seis transectos, establecidos a 5, 10, 15 y $20 \mathrm{~m}$ de profundidad (paralelo a la línea de costa), en una zona protegida del oleaje ( $7.5 \mathrm{~m}$ de profundidad) y en un sitio expuesto al oleaje ( $10 \mathrm{~m}$ de profundidad). La abundancia total de cangrejos fue similar entre bahías. Se observó una correlación positiva entre la presencia de individuos inmaduros, hembras adultas y hembras ovígeras con la temperatura del fondo y superficial, mientras que los machos adultos mostraron una tendencia opuesta con respecto a estos factores. La mayor abundancia de individuos inmaduros y pequeños fue detectada en los transectos protegidos y menos profundos. Los machos fueron más abundantes en los transectos menos profundos, y las hembras a 15 y $20 \mathrm{~m}$ de profundidad. Este estudio reveló que los diferentes grupos demográficos ocupan el hábitat de diferentes maneras, de acuerdo con las características locales. En general, el sureste de Brasil ofrece diferentes condiciones que favorecen la reproducción y consecuentemente la continuidad y el crecimiento de la población local de $C$. ornatus, contribuyendo a la relativamente alta abundancia de la especie en esta zona costera.

Palabras clave: monitoreo costero, ocupación preferida, ontogenia, pesca destructiva, cangrejos nadadores.

\section{INTRODUCTION}

Portunid crabs of the genus Callinectes are highly abundant along the coast of Brazil. The distribution of Callinectes ornatus Ordway 1863 is restricted to the western Atlantic, from North Carolina to Florida, in the Gulf of Mexico and the Caribbean, and along the coasts of Colombia, Venezuela, Guyana, and Brazil (Amapá to Rio Grande) (Melo 1996).

\section{INTRODUCCIÓN}

Los cangrejos portúnidos del género Callinectes son muy abundantes en la costa de Brasil. La distribución de Callinectes ornatus Ordway 1863 se restringe al Atlántico occidental, encontrándose de Carolina del Norte a Florida, en el golfo de México y el Caribe, y en las costas de Colombia, Venezuela, Guyana y Brasil (entre Amapá y Río Grande) 
This species occurs mainly in environments with sand or mud bottoms and less-saline waters, and can reach a depth of $75 \mathrm{~m}$ in some areas (Melo 1996). Maximum abundance of C. ornatus in the Ubatuba region (Brazil) was found at 5 to $15 \mathrm{~m}$ depth, and the abundance decreases significantly toward $25 \mathrm{~m}$ (Bertini and Fransozo 2004). It is unknown, however, if the abundances of male and female crabs differ in relation to depth.

Habitat selection by swimming crabs depends on the particular physiological requirements of each stage of their life cycle (Guillory et al. 2001). Blue crabs have planktonic, nektonic, and benthic stages, with offshore marine to nearshore estuarine phases (Guillory et al. 2001). In some species of the genus Callinectes, immature females that have not reached their pubertal molt seek out low-salinity zones of estuaries with high densities of mature males for mating (Guillory et al. 2001). Juvenile females molt and copulate in the brackish waters of the upper estuary (Johnson and Perry 1999). After successful mating, females migrate out of the estuaries for the purpose of larval dispersal.

The distribution patterns of blue crabs seem to be a result of habitat preferences combined with intra- and interspecific interactions (Buchanan and Stoner 1988). The different demographic groups within a species have different distribution patterns. For instance, ovigerous females of the genus Callinectes display a cryptic habit in order to protect their offspring, thus preferring waters with higher salinity (Pita et al. 1985, Mantelatto 2000). Females of C. ornatus can be found farther off the coast than females of the congener Callinectes danae, since the larvae of $C$. ornatus are less tolerant to variations in salinity and temperature (NegreirosFransozo et al. 1999). Keunecke et al. (2012), studying the reproductive strategies of two sympatric species of Callinectes in an estuarine system, suggested that ovigerous females of $C$. ornatus may occupy the ecotone areas nearer the open sea.

To investigate the hypothesis of differential occupation of space and depths by demographic groups of $C$. ornatus, their spatial and temporal distribution and sex ratio were studied, taking environmental factors into consideration. This information is important, not only to elucidate the biology of the species, but also to assist in environmental management, particularly in relation to the delimitation of marine protected areas.

\section{MATERIALS AND METHODS}

\section{Field collections}

Swimming crabs were collected monthly from January 1998 to December 1999 at Ubatumirim, Ubatuba, and Mar Virado bays on the southeastern Brazilian coast $\left(23^{\circ} 35^{\prime} 00^{\prime \prime} \mathrm{S}\right.$, $\left.45^{\circ} 12^{\prime} 30^{\prime \prime} \mathrm{W} ; 23^{\circ} 22^{\prime} 30^{\prime \prime} \mathrm{S}, 44^{\circ} 53^{\prime} 24^{\prime \prime} \mathrm{W}\right)$. Six transects were established in each bay, at 5,10,15, and $20 \mathrm{~m}$ depth (parallel to the beach line), in an area protected from wave action (7.5 $\mathrm{m}$ depth), and at an exposed site (10 $\mathrm{m}$ depth), the latter
(Melo 1996). Esta especie habita principalmente en ambientes con fondos arenosos o lodosos y aguas menos salinas, y puede alcanzar una profundidad de $75 \mathrm{~m}$ en algunas zonas (Melo 1996). En la región de Ubatuba (Brasil), la máxima abundancia de $C$. ornatus ha sido registrada a profundidades de $5 \mathrm{a} 15 \mathrm{~m}$, su abundancia disminuyendo considerablemente hacia los $25 \mathrm{~m}$ de profundidad (Bertini y Fransozo 2004); sin embargo, se desconoce si la abundancia de machos y hembras difiere en relación con la profundidad.

La selección de hábitat por cangrejos nadadores depende de los requerimientos fisiológicos particulares de cada etapa de su ciclo de vida (Guillory et al. 2001). Los cangrejos azules tienen etapas planctónicas, nectónicas y bentónicas, con fases estuarinas y oceánicas (Guillory et al. 2001). Las hembras inmaduras de algunos miembros del género Callinectes que no han alcanzado la muda de pubertad buscan zonas estuarinas de baja salinidad con altas densidades de machos maduros para su apareamiento (Guillory et al. 2001). La muda y el apareamiento de hembras jóvenes se realiza en aguas salobres de las partes altas de estuarios (Johnson y Perry 1999). Después de un apareamiento exitoso, las hembras migran de los estuarios al mar abierto para la dispersión de sus larvas.

Los patrones de distribución de los cangrejos azules parecen estar relacionados con la preferencia de hábitat en conjunto con interacciones intra e interespecíficas (Buchanan y Stoner 1988). Los diversos grupos demográficos de una especie tienen diferentes patrones de distribución. Por ejemplo, las hembras ovígeras del género Callinectes presentan hábitos crípticos para proteger a sus crías y prefieren aguas de mayor salinidad (Pita et al. 1985, Mantelatto 2000). Las hembras de $C$. ornatus pueden encontrarse a mayor distancia de la costa que las hembras de su congénere Callinectes danae, ya que las larvas de C. ornatus son menos tolerantes a las variaciones de salinidad y temperatura (Negreiros-Fransozo et al. 1999). Al estudiar las estrategias reproductivas de dos especies simpátricas de Callinectes, Keunecke et al. (2012) observaron que las hembras ovígeras de C. ornatus pueden ocupar las zonas de ecotonía más cercanas al mar abierto.

Para examinar la hipótesis de la ocupación diferencial del espacio y la profundidad por diferentes grupos demográficos de C. ornatus, se estudiaron su distribución espacial y temporal y la proporción de sexos, teniendo en cuenta los factores ambientales. Esta información es importante, no sólo para dilucidar la biología de la especie, sino también para apoyar la gestión ambiental, particularmente en lo referente a la delimitación de áreas marinas protegidas.

\section{MATERIALES Y MÉTODOS}

\section{Trabajo de campo}

Se recolectaron especímenes de C. ornatus mensualmente entre enero de 1998 y diciembre de 1999 en las bahías de 
two near rocky shores and perpendicular to the shoreline (fig. 1a). The characteristics of the exposed and sheltered areas were determined according to the position of the transect in relation to exposure to wave action from the ocean currents, which flow from north to south. The crabs were collected from a shrimp fishing boat equipped with doublerig nets with the following net specifications: $11 \mathrm{~m}$ in length, $4.5 \mathrm{~m}$ at the mouth, $25 \mathrm{~mm}$ body mesh diameter, and $15 \mathrm{~mm}$ cod-end mesh diameter. Each transect was trawled over a 30 -min period, covering an area of $18,000 \mathrm{~m}^{2}$ at a speed of $2.1 \mathrm{mph}$.

\section{Environmental variables}

For each transect, the bottom and surface water temperatures were recorded using a thermometer attached to a Nansen bottle. An ecobathymeter coupled with a GPS was used to record depths at the sampling sites. Sediment samples were collected in each season with a Van Veen grab, sampling a bottom area of $0.06 \mathrm{~m}^{2}$ in order to measure the organic-matter content and grain size of the sediments. In the laboratory, the sediment was oven-dried at $72{ }^{\circ} \mathrm{C}$ for $72 \mathrm{~h}$. The sediment organic-matter content (\%) was obtained by ash-weighing: three aliquots of $10 \mathrm{~g}$ each per station were placed in porcelain crucibles, incinerated for $3 \mathrm{~h}$ at $500{ }^{\circ} \mathrm{C}$, and the ashes were weighed (Mantelatto and Fransozo 1999). Sediment grain-size composition (phi) was analyzed according to Bertini and Fransozo (2004). The sediment remaining after analysis of the organic matter was re-dried and passed through a series of sieves with graduated mesh sizes, following the Wentworth (1922) scale.
Ubatumirim, Ubatuba y Mar Virado en el sureste de Brasil $\left(23^{\circ} 35^{\prime} 00^{\prime \prime} \mathrm{S}, 45^{\circ} 12^{\prime} 30^{\prime \prime} \mathrm{W} ; 2^{\circ} 22^{\prime} 30^{\prime \prime} \mathrm{S}, 44^{\circ} 53^{\prime} 24^{\prime \prime} \mathrm{W}\right)$. En cada bahía se establecieron seis transectos, a 5, 10, 15 y $20 \mathrm{~m}$ de profundidad (paralelo a la línea de costa), en una zona protegida del oleaje ( $7.5 \mathrm{~m}$ de profundidad) y en una zona expuesta al oleaje (10 $\mathrm{m}$ de profundidad), los últimos dos cerca de costas rocosas y perpendicular a la línea de costa (fig. 1a). Las características de la zona protegida y la zona expuesta se determinaron según la posición del transecto en relación al oleaje proveniente de las corrientes oceánicas, que fluyen de norte a sur. Los cangrejos fueron recolectados desde un barco camaronero equipado con dos redes de arrastre (11 m de largo, $4.5 \mathrm{~m}$ de boca, $25 \mathrm{~mm}$ de luz de malla, $15 \mathrm{~mm}$ de luz de malla en el copo). Cada transecto se recorrió durante $30 \mathrm{~min}$, cubriendo un área de $18,000 \mathrm{~m}^{2} \mathrm{a}$ una velocidad de $2.1 \mathrm{mph}$.

\section{Variables ambientales}

En cada transecto se registró la temperatura superficial y del fondo con un termómetro instalado en una botella Nansen. Se usó un ecobatímetro con un sistema de posicionamiento global para registrar las profundidades en los sitios de muestreo. Se recolectaron muestras de sedimento en cada temporada con una draga Van Veen, muestreando un área de $0.06 \mathrm{~m}^{2}$ para medir el contenido de materia orgánica y el tamaño de grano de los sedimentos. En el laboratorio, las muestras se secaron en un horno a $72{ }^{\circ} \mathrm{C}$ durante $72 \mathrm{~h}$. Para determinar el contenido de materia orgánica (\%), se colocaron tres alícuotas de $10 \mathrm{~g}$ cada una de cada estación en crisoles de porcelana, las muestras se incineraron durante $3 \mathrm{~h}$ a
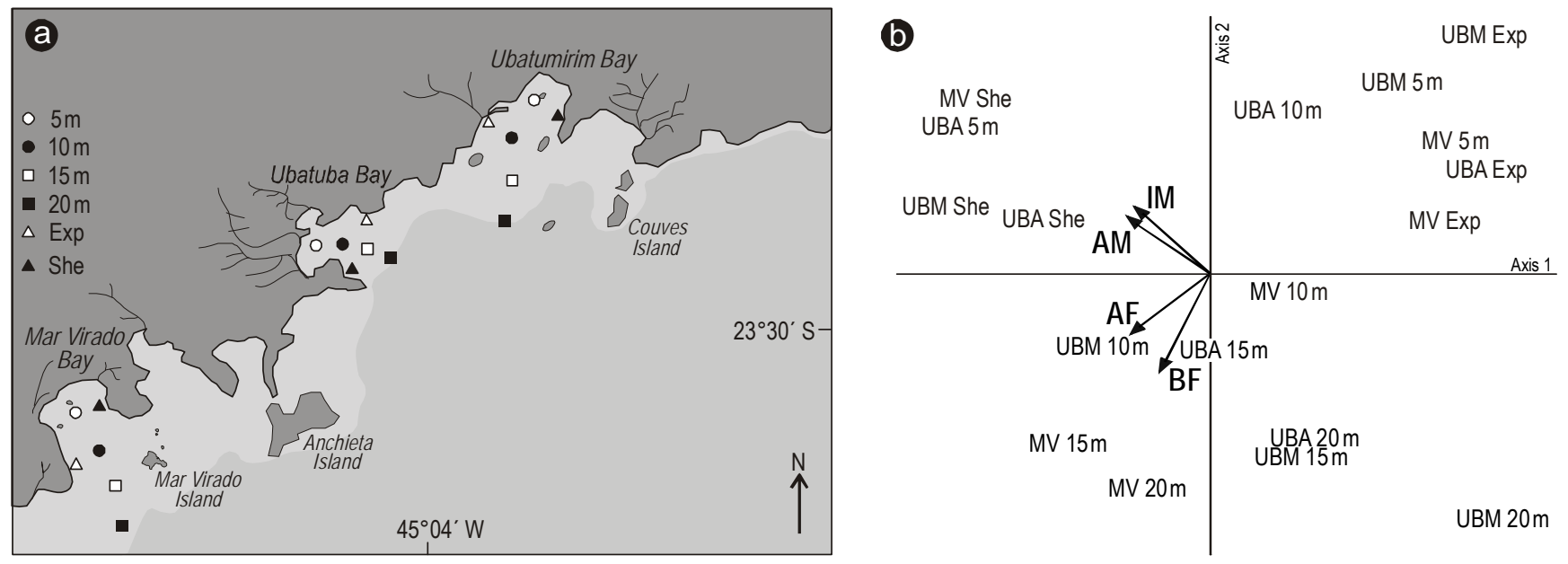

Figure 1. Differential distribution of the demographic groups of Callinectes ornatus. (a) The three bays and their respective transects. (b) Ordination of the four demographic groups and the transects in each bay according to a principal components analysis. IM, immature individuals; AM, adult males; AF, non-breeding adult females; BF, breeding females; UBM, Ubatumirim Bay; UBA, Ubatuba Bay; MV, Mar Virado Bay; She, wave-sheltered area; Exp, wave-exposed area.

Figura 1. Distribución diferencial de los grupos demográficos de Callinectes ornatus. (a) Las tres bahías y sus transectos respectivos. (b) Ordenación de los cuatro grupos demográficos y los transectos en cada bahía según el análisis de componentes principales. IM, individuos inmaduros; AM, machos adultos; AF, hembras adultas sin huevos; BF, hembras ovígeras; UBM, bahía de Ubatumirim; UBA, bahía de Ubatuba; MV, bahía de Mar Virado; She, zona protegida del oleaje; Exp, zona expuesta al oleaje. 


\section{Laboratory procedure}

Individuals of C. ornatus were identified (Melo 1996) and separated by bay, transect, and collection month. All crabs were sexed by examining the abdominal morphology. The adherence of the abdomen to the thoracic sternites was checked to identify immature individuals (Mantelatto and Fransozo 1999) and to assess the size at sexual maturity. For females, the presence of eggs on the pleopods was also observed, and those that carried embryonated eggs attached to their pleopods were considered breeding females. Thus, the crabs were separated into four demographic categories: adult males, non-breeding adult females, breeding females, and immature individuals. The absolute frequency of these categories was calculated based on the total number of individuals recorded along each transect in each month. Each crab was measured for carapace length and width without the lateral teeth, using a caliper $(0.01 \mathrm{~mm})$.

\section{Statistical analyses}

Abundance data were log-transformed, and a one-way analysis of variance (ANOVA) was carried out to assess differences in the total abundance of individuals among bays $(\alpha=0.05)$ (Zar 1996). To investigate the temporal variation in the abundance of each demographic group, a correspondence analysis was conducted, followed by a multi-response permutation procedure (MRPP) test. In the correspondence analysis, the percentage of explanation is obtained by dividing the eigenvalue by the total inertia. A principal components analysis (PCA) was used to identify the relationship between environmental variables (bottom temperature, salinity, organic matter, and phi) and the 18 transects and between the four demographic groups and the transects in each bay. The axes (determined by the broken-stick cut-off criterion, following McCune and Grace 2002) of the "environment vs transects" PCA were used as the environmental matrix in a canonical correspondence analysis (CCA), in which the biotic matrix was represented by the abundance of different demographic groups by transect.

Coordination points were calculated using linear combination scores (fitted values from the weighted averaging regression of the species scores; see McCune and Grace 2002), and the relative contributions of variables on each axis were determined by the intraset correlation (McCune and Grace 2002). The statistical significances of the eigenvalues and the species vs environment correlations were evaluated by randomization (Monte Carlo tests), using 1000 randomized runs for each analysis. In each randomization, sample units in the environmental matrix were shuffled. This destroys the relationship between the species and environmental matrices, while preserving the species matrix and the correlation structure of the environmental matrix. All multivariate analyses were carried out using the software PC-ORD 6.0 , conforming to all assumptions of normality.
$500{ }^{\circ} \mathrm{C}$, y las cenizas se pesaron (Mantelatto y Fransozo 1999). El tamaño de grano (phi) se analizó de acuerdo con Bertini y Fransozo (2004). Después del análisis de la materia orgánica, el sedimento restante se volvió a secar y se pasó a través de una serie de tamices con luz de malla decreciente, según la escala de Wentworth (1922).

\section{Trabajo de laboratorio}

Los individuos de C. ornatus fueron identificados (Melo 1996) y separados por bahía, transecto y mes de recolección. Se examinó la morfología del abdomen para determinar el sexo de todos los cangrejos. Se revisó la adherencia del abdomen a los esternitos torácicos para identificar los individuos inmaduros (Mantelatto y Fransozo 1999) y evaluar la talla de madurez sexual. En el caso de las hembras, también se observó la presencia de huevos en los pleópodos, y las que presentaron huevos embrionados adheridos a los pleópodos se consideraron hembras ovígeras. Así, los cangrejos fueron separados en cuatro grupos demográficos: machos adultos, hembras adultas sin huevos, hembras ovígeras e individuos inmaduros. La frecuencia absoluta de estas categorías se calculó con base en el número total de individuos registrados en cada transecto en cada mes. Se midieron la longitud y el ancho del caparazón, sin los dientes laterales, de cada cangrejo con un calibrador ( $0.01 \mathrm{~mm}$ de precisión).

\section{Análisis estadísticos}

Los datos de abundancia fueron transformados logarítmicamente, realizándose un análisis de varianza (ANOVA, por sus siglas en inglés) de una vía para evaluar las diferencias en la abundancia total de individuos entre bahías $(\alpha=$ 0.05) (Zar 1996). Para examinar la variación temporal de la abundancia de cada grupo demográfico, se realizó un análisis de correspondencias, seguido por una prueba de permutación (procedimientos de multirespuesta por permutación, MRPP). En el análisis de correspondencias, el porcentaje de explicación se obtiene dividiendo el valor propio por la inercia total. Se realizó un análisis de componentes principales (PCA) para identificar la relación entre las variables ambientales (temperatura del fondo, salinidad, materia orgánica y phi) y los 18 transectos, así como la relación entre la abundancia de los cuatro grupos demográficos y los transectos en cada bahía. Los ejes (determinados mediante el criterio de limitación [broken-stick cut-off criterion] según McCune y Grace 2002) de la relación entre transectos y ambiente del PCA fueron usados como la matriz ambiental en un análisis de correspondencias canónico (CCA), en el cual la matriz biótica fue representada por la abundancia de los diferentes grupos demográficos por transecto.

Se calcularon los puntos de coordinación con los puntos de combinaciones lineales (valores ajustados de la regresión ponderada de las puntuaciones de las especies; ver McCune y Grace 2002), y se determinaron las contribuciones relativas 
The mean carapace width $(\mathrm{mm})$ was tested for normality and homoscedasticity through the Shapiro-Wilks and Levene tests, and was compared between males and females using a Mann-Whitney test (the significance level adopted was 5\%). To assess the structure of the population according to bays and transects, a factorial ANOVA (bays vs transects vs within-group carapace width) was performed, and Tukey's multiple comparison tests were carried out to analyze for statistical differences between means at the 5\% significance level. The male to female sex ratio, by season and transects, was compared by the binomial test (Wilson and Hardy 2002).

\section{RESULTS}

\section{Distribution, abundance, and environmental variables}

A total of 20,521 specimens, including 6464 adult males, 5036 adult females (871 breeding), and 9021 immature individuals, were analyzed. Although the total abundance of crabs among the bays was similar (ANOVA; $F=0.32 ; P=$ 0.73 ), the ordination analysis of demographic groups by transects indicated an ontogenetic shift toward deeper waters (fig. 1b). This shift was most evident in the case of females, whereas juveniles were most commonly found in shallow water near rocky shores (fig. 1b).

The overall abundance of individuals oscillated over time (fig. 2). Immature individuals and females showed a peak in abundance during the warmer months, except in Ubatumirim, where the peak abundance of immature individuals occurred in midwinter of 1998. Adult males were more abundant during the colder months (fig. 2). This fluctuation was detected by reciprocal averaging, which indicated a positive correlation between the abundance of adult and breeding females with warmer months, and between adult males and months with lower temperatures. Immature individuals and breeding females were negatively associated with low-temperature months (table 1). These results were confirmed by MRPP (average within-group) (table 1).

During the study period, the widest variations in bottom temperature occurred in 1998, when significant differences were observed among transects, as detected by PCA. Similar differences in salinity occurred in 1999, varying among transects and also among bays. In general, silt and clay proportions ( $\mathrm{C}$ fraction), phi values, and organic-matter content (\%) increased from north to south in the different bays. Within each bay, the lowest proportions of silt and clay were observed at $20 \mathrm{~m}$ depth, with an increase toward shallower transects (fig. 3a).

The PCA indicated that the environmental variables (bottom temperature, salinity, organic matter, and phi), as well as the demographic groups (adult males, non-breeding adult females, breeding females, and immature individuals), were strongly correlated among transects (table 2 ; figs. $1 \mathrm{~b}, 3 \mathrm{~b}$ ). In the ordination plots, variables are represented as arrows, and their length indicates the relative importance of each de las variables en cada eje mediante la correlación intragrupo (McCune y Grace 2002). La significación estadística de los valores propios y las correlaciones especie vs ambiente se evaluaron mediante un proceso de aleatorización (pruebas Monte Carlo), con 1000 corridas aleatorias para cada análisis. En cada aleatorización, se mezclaron las unidades de muestreo en la matriz ambiental. Esto destruye la relación entre las matrices ambiental y biótica, pero preserva la matriz biótica y la estructura de correlación de la matriz ambiental. Todos los análisis multivariantes se realizaron con el programa PC-ORD 6.0, cumpliendo con todos los supuestos de normalidad.

Se comprobó la normalidad y homocedasticidad del ancho de caparazón $(\mathrm{mm})$ promedio mediante las pruebas de Shapiro-Wilks y Levene, y los datos para hembras y machos fueron comparados con una prueba de Mann-Whitney (a un nivel de significación de 5\%). Para evaluar la estructura de la población en cuanto a bahías y transectos, se realizó un ANOVA factorial (bahías vs transectos vs ancho de caparazón intragrupo), y se llevaron a cabo pruebas de comparaciones múltiples de Tukey para analizar las diferencias estadísticas entre los promedios a un nivel de significación de $5 \%$. La proporción de machos y hembras, por estación del año y transecto, se comparó mediante una prueba binomial (Wilson y Hardy 2002).

\section{RESUlTADOS}

\section{Distribución, abundancia y variables ambientales}

Se analizaron 20,521 especímenes, de los cuales 6464 fueron machos adultos, 5036 fueron hembras adultas (871 ovígeras) y 9021 fueron individuos inmaduros. Aunque la abundancia total de $C$. ornatus entre las bahías fue similar (ANOVA; $F=0.32 ; P=0.73$ ), el análisis de ordenación de los grupos demográficos por transecto mostró un cambio ontogenético hacia aguas más profundas (fig. 1b). Este cambio fue más evidente en el caso de las hembras, mientras que los individuos inmaduros comúnmente se encontraron en agua somera cerca de costas rocosas (fig. 1b).

La abundancia de individuos varió temporalmente (fig. 2). Los individuos inmaduros y las hembras fueron más abundantes durante los meses más cálidos, excepto en Ubatumirim, donde la máxima abundancia de los primeros se registró a mediados del invierno de 1998. Los machos adultos fueron más abundantes en los meses más fríos. Esta fluctuación se determinó mediante el análisis de promedios recíprocos, el cual indicó una correlación positiva entre la abundancia de hembras adultas y ovígeras con los meses más cálidos, y entre los machos adultos y los meses más fríos. Los individuos inmaduros y las hembras ovígeras presentaron una asociación negativa con los meses fríos (tabla 1). Estos resultados fueron confirmados mediante MRPP (promedio intragrupo) (tabla 1). 

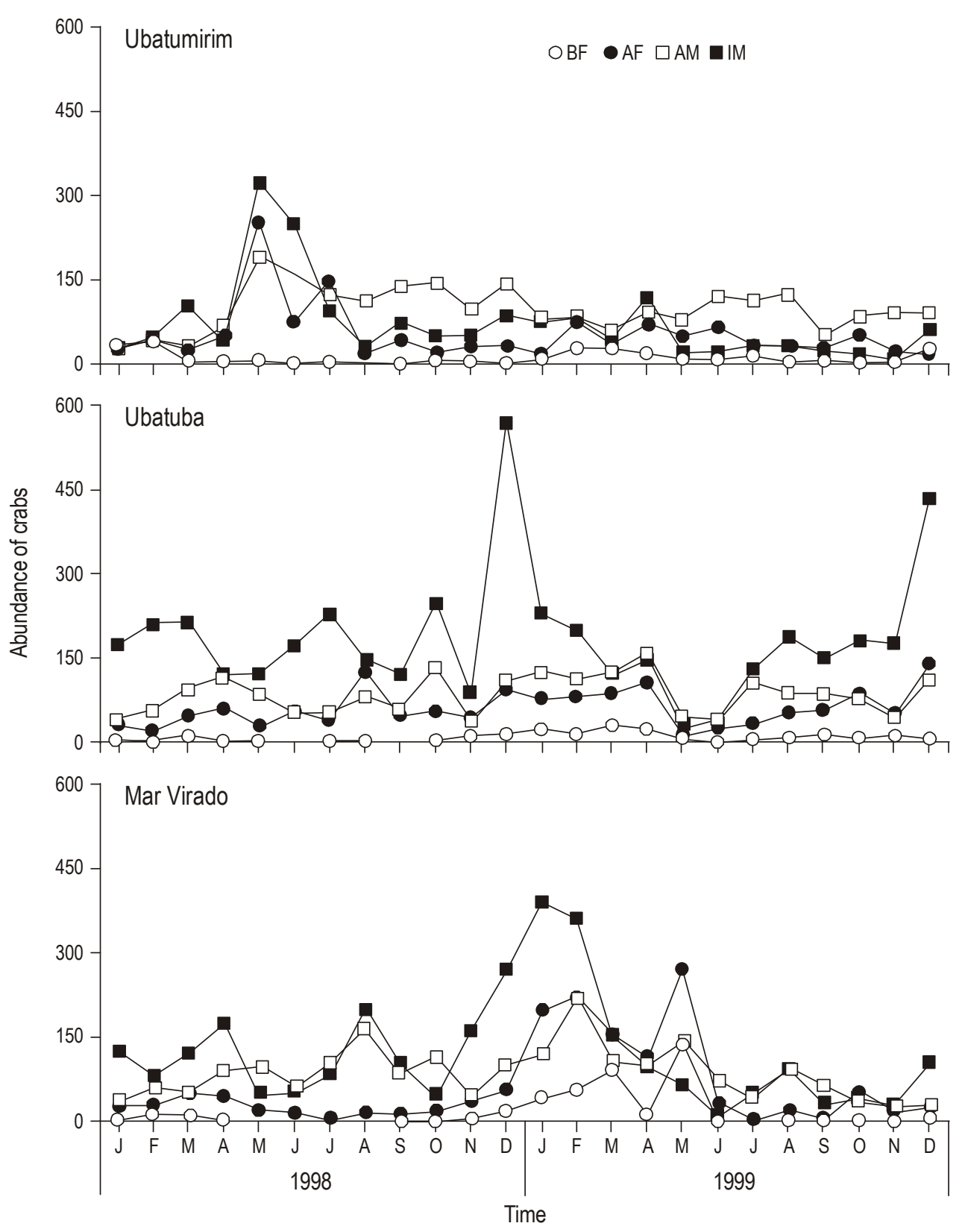

Figure 2. Monthly abundance of the demographic groups of Callinectes ornatus in Ubatumirim, Ubatuba, and Mar Virado bays, during the period from January 1998 to December 1999. IM, immature individuals; AM, adult males; AF, non-breeding adult females; BF, breeding females.

Figura 2. Abundancia mensual de los grupos demográficos de Callinectes ornatus en las bahías de Ubatumirim, Ubatuba y Mar Virado, durante el periodo de enero de 1998 a diciembre de 1999. IM, individuos inmaduros; AM, machos adultos; AF, hembras adultas sin huevos; $\mathrm{BF}$, hembras ovígeras.

variable. The angle between arrows represents the degree of correlation between the corresponding environmental variables, whereas the location of species in relation to the arrow indicates the characteristics of the locations and preferences of the species. This result demonstrates that the transects can be characterized by their environmental variables, as well as the demographic groups. The CCA revealed two axes of influence of environmental factors on the distribution of
Durante el periodo de estudio, las mayores variaciones de la temperatura del fondo se registraron en 1998, y el PCA mostró diferencias significativas entre los transectos. En 1999 la salinidad mostró diferencias similares, variando entre transectos así como entre bahías. En general, las proporciones de limo y arcilla (fracción C), los valores de phi y el contenido de materia orgánica (\%) incrementaron de norte a sur en las bahías. Dentro de cada bahía, las proporciones 
Table 1. Summary of the correspondence analysis and average within-group distance (multi-response permutation procedure) between months of the year and abundance of demographic groups of Callinectes ornatus. Axis values indicate scores (weights) in relation to the ranked sampling units. $\mathrm{BF}$, breeding females; AF, non-breeding adult females; AM, adult males; IM, immature individuals.

Tabla 1. Resumen del análisis de correspondencia y de la distancia promedio intragrupos (procedimiento de multirespuesta por permutación) entre los meses del año y la abundancia de los grupos demográficos de Callinectes ornatus. Los valores de los ejes indican puntuaciones (ponderaciones) en relación con las unidades de muestreo ordenadas. $\mathrm{BF}$, hembras ovígeras; $\mathrm{AF}$, hembras adultas sin huevos; AM, machos adultos; IM, individuos inmaduros.

\begin{tabular}{|c|c|c|c|}
\hline \multicolumn{4}{|c|}{ Correspondence analysis } \\
\hline Group & Axis 1 & Group & Axis 2 \\
\hline $\mathrm{BF}$ & 331 & $\mathrm{AM}$ & 139 \\
\hline $\mathrm{AF}$ & 156 & $\mathrm{AF}$ & 16 \\
\hline AM & -39 & IM & -94 \\
\hline IM & -75 & $\mathrm{BF}$ & -134 \\
\hline \multicolumn{2}{|c|}{ Ranked 1} & \multicolumn{2}{|c|}{ Ranked 2} \\
\hline Jan 1999 & 201 & May 1998 & 108 \\
\hline May 1999 & 167 & Jul 1998 & 106 \\
\hline Nov 1999 & 145 & Oct 1998 & 91 \\
\hline May 1999 & 144 & Jun 1999 & 89 \\
\hline Dec 1999 & 142 & Jul 1999 & 87 \\
\hline Jan 1998 & 138 & Aug 1999 & 87 \\
\hline Nov 1999 & 121 & Jun 1999 & 82 \\
\hline Nov 1998 & 121 & Oct 1998 & 77 \\
\hline Sep 1999 & 106 & Jul 1999 & 75 \\
\hline Mar 1998 & 105 & Sep 1998 & 75 \\
\hline Oct 1999 & 104 & Jul 1998 & 73 \\
\hline Feb 1999 & 102 & Jun 1999 & 72 \\
\hline Dec 1998 & 100 & Oct 1999 & 72 \\
\hline Mar 1999 & 94 & May 1999 & 66 \\
\hline Feb 1998 & 92 & Nov 1999 & 56 \\
\hline \multirow[t]{2}{*}{ Inertia } & & \multicolumn{2}{|c|}{0.34} \\
\hline & & Axis 1 & Axis 2 \\
\hline Eigenvalue & & 0.21 & 0.08 \\
\hline$\%$ Variance & & 61.76 & 23.53 \\
\hline \multicolumn{4}{|c|}{ Average within-group distance } \\
\hline Group & & Average & $P$ \\
\hline $\mathrm{BF}$ & & 0.05 & 0 \\
\hline $\mathrm{AF}$ & & 0.09 & 0 \\
\hline AM & & 0.10 & 0 \\
\hline IM & & 0.09 & 0 \\
\hline
\end{tabular}

de limo y arcilla fueron menores a $20 \mathrm{~m}$ de profundidad e incrementaron a menor profundidad (fig. 3a).

El PCA mostró que tanto las variables ambientales (temperatura del fondo, salinidad, materia orgánica y phi) como los grupos demográficos (machos adultos, hembras adultas sin huevos, hembras ovígeras e individuos inmaduros) se correlacionaron fuertemente entre transectos (tabla 2; figs. 1b, 3b). En las gráficas de ordenación, las variables se representan con flechas y su longitud indica la importancia relativa de cada variable. El ángulo entre las flechas representa el grado de correlación entre las variables ambientales correspondientes, mientras que la ubicación de las especies en relación con la flecha indica las características de los sitios y las preferencias de las especies. Este resultado demuestra que los transectos pueden ser caracterizados por sus variables ambientales, así como por los grupos demográficos. El CCA mostró dos ejes de influencia de los factores ambientales en la distribución de C. ornatus, con una varianza (inercia) total de $12.47 \%$ (tabla 3 ). La correlación entre los grupos demográficos de los dos ejes canónicos fue estadísticamente significativa (prueba de permutación Monte Carlo, $P<$ 0.001 ), lo que indica una correspondencia entre los grupos demográficos y las variables predictoras (tabla 3). Estos ejes canónicos explicaron el $17.1 \%$ de la variación en la distribución de los grupos demográficos de C. ornatus. Las hembras ovígeras contribuyeron altamente a esta variación, ya que fue el grupo que mejor se correlacionó con el eje. La presencia de individuos inmaduros mostró una relación positiva con el tamaño de grano (phi). La fuerza y dirección de las relaciones entre las variables predictoras y los grupos demográficos se muestran en la tabla 4.

\section{Estructura de la población y proporción de sexos}

La distribución diferencial de machos y hembras se confirmó mediante una prueba binomial, que mostró una mayor proporción de hembras a $15 \mathrm{~m}$ (media de las bahías = $1 \mathrm{M}: 1.4 \mathrm{H} ; P<0.01$ ) y $20 \mathrm{~m}$ de profundidad (media de las bahías $=1 \mathrm{M}: 2.3 \mathrm{H} ; P<0.01)$. En los otros transectos, la proporción de machos fue mayor o la proporción de sexos fue igual. En otoño de 1999, las hembras fueron más abundantes que los machos $(1 \mathrm{M}: 1.1 \mathrm{H}, P<0.01)$, mientras que en el verano del mismo año, la proporción de sexos fue igual (1M:1H, $P=0.3)$. En las otras estaciones del año, los machos predominaron $(P<0.05)$.

En general, la estructura de tallas de la población fue estable. La longitud media ( \pm desviación estándar) del caparazón de C. ornatus fue $61.24 \pm 1.2 \mathrm{~mm}$ para los machos adultos, $51.30 \pm 1.2 \mathrm{~mm}$ para las hembras ovígeras, $50.97 \pm 0.50 \mathrm{~mm}$ para las hembras adultas y $35.50 \pm 1.63 \mathrm{~mm}$ para los individuos inmaduros. La longitud del caparazón de todos los individuos no varió en relación con la profundidad o exposición al oleaje. El ancho de caparazón (AC) promedio para toda la población fue $47.4 \pm 13.7 \mathrm{~mm}$. Todos los individuos se agruparon en 14 clases de talla de $6 \mathrm{~mm}$ cada uno. La distribución 

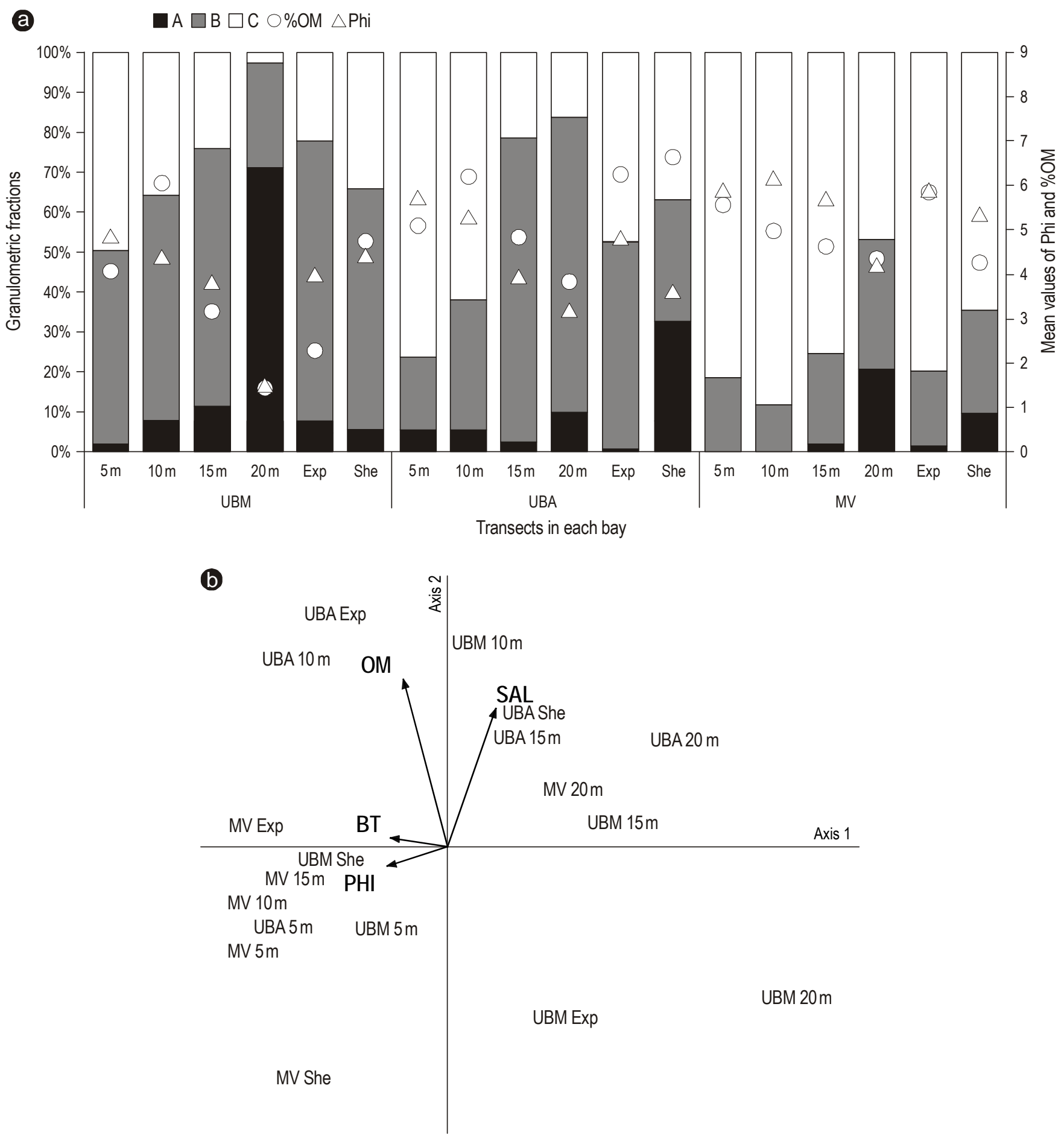

Figure 3. (a) Characterization of the transects. Proportion of grain-size classes (A, B, C), central tendency of bottom sediments (phi), and mean organic-matter content (\% OM) for each transect. A is gravel, very coarse sand, coarse sand, and medium sand; B is fine and very fine sand; and $\mathrm{C}$ is silt and clay. (b) Ordination of the four environmental variables and the transects in each bay, as defined by the principle components analysis, using linear combination scores. BT, bottom temperature; SAL, salinity; UBM, Ubatumirim Bay; UBA, Ubatuba Bay; MV, Mar Virado Bay; She, wave-sheltered area; Exp, wave-exposed area.

Figura 3. (a) Caracterización de los transectos. Proporción de las clases de tamaño de grano (A, B, C), tendencia central de los sedimentos del fondo (phi) y contenido promedio de materia orgánica $(\% \mathrm{OM})$ para cada transecto. A representa grava, arena muy gruesa, arena gruesa y arena media; B, arena fina y muy fina; y C, limo y arcilla. (b) Ordenación de las cuatro varibales ambientales y los transectos en cada bahía, según el análisis de componentes principales, usando puntos de combinaciones lineales. BT, temperatura del fondo; SAL, salinidad; UBM, bahía de Ubatumirim; UBA, bahía de Ubatuba; MV, bahía de Mar Virado; She, zona protegida del oleaje; Exp, zona expuesta al oleaje. 
C. ornatus, with a total variance (inertia) of $12.47 \%$ (table 3 ). The correlation among the demographic groups and the two canonical axes was statistically significant (Monte-Carlo permutation test, $P<0.001$ ), indicating correspondence between the demographic groups and the predictor variables (table 3). These canonical axes explained $17.1 \%$ of the variation in the distribution of the demographic groups of $C$. ornatus. Breeding females made a high contribution to this variation, since it was the group that better correlated with the axis. The occurrence of immature individuals showed a positive relationship with mean grain size (phi). The strength and direction of the relationships between the predictor variables and demographic groups are shown in the CCA scores and biplots (table 4).

\section{Population structure and sex ratio}

The differential distribution between genders was confirmed by a binomial test, which indicated higher proportions of females at $15 \mathrm{~m}$ (mean of bays $=1 \mathrm{M}: 1.4 \mathrm{~F} ; P<0.01$ ) and $20 \mathrm{~m}$ depth (mean of bays $=1 \mathrm{M}: 2.3 \mathrm{~F} ; P<0.01$ ). Along the other transects, the sex ratio favored males or showed equal proportions. Females were more abundant than males (1M:1.1F, $P<0.01)$ in autumn 1999, whereas in summer 1999 this proportion was the same $(1 \mathrm{M}: 1 \mathrm{~F}, P=0.3)$. During the other seasons, males predominated $(P<0.05)$.

Generally, the size structure of the population was stable. The mean ( \pm standard deviation) length of the $C$. ornatus carapace was $61.24 \pm 1.2 \mathrm{~mm}$ in adult males, $51.30 \pm 1.2 \mathrm{~mm}$ in breeding females, $50.97 \pm 0.50 \mathrm{~mm}$ in adult females, and $35.50 \pm 1.63 \mathrm{~mm}$ in immature individuals. There were no fluctuations in carapace length in relation to depth or the wave exposure site for all individuals. The mean carapace width (CW) for the entire population was $47.4 \pm 13.7 \mathrm{~mm}$. All individuals were grouped in 14 size classes of $6 \mathrm{~mm}$ each. The size-frequency distribution was normal; males were significantly larger than females (Mann-Whitney; $Z=79.3 ; P<$ 0.05 ). The size of specimens ranged from $5.1 \mathrm{~mm} \mathrm{CW}$ (an immature female caught in Ubatuba) to $84.0 \mathrm{~mm} \mathrm{CW}$ (an adult male caught in Mar Virado). Males predominated in the smallest size classes, followed by a balance between the numbers of males and females, next a predominance of females, and again a predominance of males in the $50 \mathrm{~mm}$ $\mathrm{CW}$ and larger size classes. The largest female, captured in Ubatuba, measured $67.6 \mathrm{~mm} \mathrm{CW}$. When comparing the $\mathrm{CW}$ of each age group among regions and transects, it became evident that there were significant differences in the size of immature individuals and adult males among transects. The smallest adult males were found along the transect at $20 \mathrm{~m}$ depth in Ubatumirim $(59.04 \pm 4.27 \mathrm{~mm})$ and Ubatuba $(59.14 \pm 5.36 \mathrm{~mm})$ (factorial ANOVA: $F=5.20$; $\mathrm{MS}=3.4$; $P<0.01)$; yet the smallest immature individuals were found along the sheltered transects in Ubatumirim (34.84 \pm $9.46 \mathrm{~mm})$ and Ubatuba (32.13 $\pm 7.28 \mathrm{~mm}$ ) (factorial ANOVA: $F=2.74$; MS $=19.98 ; P<0.01)$. Breeding females
Table 2. Summary statistics of the axes generated by the principal components analysis (PCA). Correlation between environmental variables and transects and between demographic groups of Callinectes ornatus and transects at Ubatumirim, Ubatuba, and Mar Virado bays. Coefficients greater than or equal to +0.4 or less than or equal to -0.4 are considered ecologically important (see Rakocinski et al. 1996) and are shown in bold. The correlation coefficients between the density of groups and the first and second PCA axes are also shown.

Tabla 2. Estadística de los ejes generados por el análisis de componentes principales. Correlación entre las variables ambientales y los transectos y entre los grupos demográficos de Callinectes ornatus y los transectos establecidos en las bahías de Ubatumirim, Ubatuba y Mar Virado. Los coeficientes mayores que o iguales a +0.4 o menores que o iguales a -0.4 se consideran ecológicamente importantes (ver Rakocinski et al. 1996) y se muestran en negritas. También se muestran los coeficientes de correlación entre la densidad de los grupos y el primer y el segundo eje.

\begin{tabular}{lcc}
\hline & Axis 1 & Axis 2 \\
\hline Environmental variables & & \\
Mean grain size & $\mathbf{- 0 . 9 6}$ & -0.07 \\
Bottom temperature & $\mathbf{- 0 . 8 9}$ & 0.01 \\
Organic matter & $\mathbf{- 0 . 7 8}$ & $\mathbf{- 0 . 5 2}$ \\
Salinity & $\mathbf{0 . 7 2}$ & $\mathbf{- 0 . 6 4}$ \\
Eigenvalue & 2.85 & 0.69 \\
\% Variance & 71.16 & 17.21 \\
\hline Demographic groups & & \\
Adult males & $\mathbf{0 . 8 9}$ & 0.33 \\
Immature individuals & $\mathbf{- 0 . 8 5}$ & $\mathbf{0 . 4}$ \\
Non-breeding adult females & $\mathbf{- 0 . 8 1}$ & $\mathbf{- 0 . 5 3}$ \\
Breeding females & -0.22 & $\mathbf{- 0 . 9 5}$ \\
Eigenvalue & 2.22 & 1.46 \\
\% Variance & 55.45 & 36.61 \\
\hline
\end{tabular}

de frecuencias de tallas fue normal; los machos fueron significativamente mayores que las hembras (Mann-Whitney; $Z=79.3 ; P<0.05)$. El tamaño de los especímenes varió de $5.1 \mathrm{~mm}$ AC (una hembra inmadura capturada en Ubatuba) a $84.0 \mathrm{~mm}$ AC (un macho adulto capturado en Mar Virado). Los machos predominaron en las clases de talla menores, luego hubo un equilibrio entre el número de machos $\mathrm{y}$ hembras, seguido por una predominancia de hembras y de nuevo una predominancia de machos en las clases de talla de $50 \mathrm{~mm}$ AC y mayores. La hembra de mayor tamaño, capturada en Ubatuba, midió $67.6 \mathrm{~mm}$ AC. Al comparar el AC de cada grupo de edad entre sitios y transectos, fue evidente que existían diferencias significativas en las tallas de los individuos inmaduros y los machos adultos entre transectos. Los machos adultos de menor tamaño se encontraron en el transecto de $20 \mathrm{~m}$ de Ubatumirim $(59.04 \pm 4.27 \mathrm{~mm}) \mathrm{y}$ Ubatuba (59.14 $\pm 5.36 \mathrm{~mm}$ ) (ANOVA factorial: $F=5.20$; $\mathrm{MS}=3.4 ; P<0.01)$, pero los individuos inmaduros más 
Table 3. Axis summary statistics of the canonical correspondence analysis and Monte Carlo test for eigenvalues and correlations between demographic groups (DG) and environmental variables (EV) based on 9999 runs with randomized data.

Tabla 3. Estadística de los ejes del análisis de correspondencias canónica y la prueba de Monte Carlo para los valores propios y las correlaciones entre los grupos demográficos (DG) y las variables ambientales (EV) con base en 9999 corridas con datos aleatorios.

\begin{tabular}{lcc}
\hline Axis summary statistics & Axis 1 & Axis 2 \\
\hline Eigenvalue & 0.019 & 0.002 \\
Variance in species data & & 2.0 \\
$\quad \%$ of variance explained & 15.1 & 17.1 \\
$\quad$ Cumulative \% explained & 15.1 & 0.294 \\
Pearson correlation, DG-EV & 0.498 & 0.205 \\
Kendall (rank) correlation DG-EV & 0.369 & \\
\hline
\end{tabular}

Monte-Carlo test

Axis $\quad$ Real data

Randomized data

$P$

\begin{tabular}{ccccc} 
Axis & Real data & \multicolumn{2}{c}{ Randomized data } & Maximum \\
\cline { 3 - 5 } & & Mean & Minimum & 0.012 \\
\hline Eigenvalue & 0.019 & 0.003 & 0.000 & 0.002 \\
1 & 0.002 & 0.000 & 0.000 & 0.366 \\
DG-EV Correlation & & & & 0.001 \\
1 & 0.498 & 0.190 & 0.029 & 0.293 \\
\hline
\end{tabular}

and adult females did not show significant differences in $\mathrm{CW}$ among bays and transects.

\section{Discussion}

The migration of breeding females to areas farther offshore seems to be a behavior to increase dispersal and maximize larval survival; however, for $C$. ornatus, this behavior was only inferred from catch records. The $C$. danae and Callinectes sapidus juveniles are euryhaline and the life stages move between seawater, estuaries and fresh water, which does not occur with $C$. ornatus. The data from this study indicated a differential use of habitat according to the requirements of the different developmental stages. Immature individules and adult males were more common in shallow areas and near rocky shores. Adult males were also abundant in areas favorable for mating (shallower depths). In contrast, breeding females migrated to deeper areas.

The mean size of sediment particles in a certain area reflects the local hydrodynamics and the geological history, which determine the sediment stratification. Silt particles only accumulate in more sheltered conditions, whereas sand particles reflect a more dynamic and high-energy environment (Pires 1992). This may explain the high abundance of immature crabs in Ubatuba Bay, where the high concentration of organic matter and quantity of silt and clay led to more available food for immature individuals, especially in the sheltered area and near the rocky shores (Mascaró et al. 2007). Santos et al. (1995) observed a higher abundance of pequeños se encontraron en los transectos protegidos de Ubatumirim (34.84 $\pm 9.46 \mathrm{~mm})$ y Ubatuba $(32.13 \pm$ $7.28 \mathrm{~mm}$ ) (ANOVA factorial: $F=2.74$; $\mathrm{MS}=19.98 ; P<$ 0.01). Las hembras adultas y ovígeras no mostraron diferencias significativas en cuanto a $\mathrm{AC}$ entre bahías y transectos.

\section{DISCUSIÓN}

La migración de hembras ovígeras hacia mar abierto parece ser un comportamiento para maximizar la dispersión y la supervivencia de las larvas; sin embargo, en el caso de C. ornatus, este comportamiento se deduce sólo de los registros de captura. Los individuos jóvenes de C. danae y Callinectes sapidus son eurihalinos y en sus diferentes etapas de vida se mueven entre agua marina, estuarios y agua dulce, lo cual no sucede con C. ornatus. Los datos de este trabajo indican un uso diferencial de hábitats según los requerimientos de las diferentes etapas de desarrollo. Los individuos inmaduros y machos adultos fueron más comunes en zonas someras y cerca de costas rocosas. Los machos adultos también fueron abundantes en las zonas favorables para el apareamiento (profundidades más someras). En contraste, las hembras ovígeras migraron a zonas más profundas.

El tamaño medio de los granos de sedimento en una cierta área refleja la hidrodinámica local y la historia geológica, que determinan la estratificación de los sedimentos. Las partículas de limo sólo se acumulan en zonas más protegidas, mientras que las partículas de arena reflejan un ambiente más 
immature individuals of Portunus spinimanus in shallow water (4 $\mathrm{m}$ deep) in Fortaleza Bay (near the study area), where crabs as small as $10 \mathrm{~mm} \mathrm{CW}$ were caught. The larger numbers of immature individuals at shallower depths in protected areas may be related to the availability of shelter and food, in addition to high productivity, which leads to high growth rates (Hines et al. 1987). In this study, the exposed transect of Ubatuba also had an abundance of immature crabs, which can be explained by the presence of several large rocks near the transect. Immature individules of some portunid species preferentially use brackish habitats to molt, possibly because of the osmotic advantages and also the lower predation rates in these locations (Hines et al. 1987). A majority of immature individuals of Persephona mediterranea occupied Ubatuba Bay, which suggested that this bay provides especially favorable conditions for them (Bertini et al. 2001). Furthermore, it has been suggested that there are ontogenic differences in the feeding strategies of Callinectes rathbunae, which may also favor the presence of immature crabs near the coast (Mascaró et al. 2007). In a study on the bathymetric distribution of crabs, Bertini and Fransozo (2004) suggested that the population of C. ornatus predominates at depths of 2 and $5 \mathrm{~m}$, where there is a low diversity of species. This distribution restricted to lower depths can be explained by an abundance of immature individuals, since small crabs survive better where there are fewer predators and better protection (Buchanan and Stoner 1988).

The greater abundance of breeding females along the transect at $20 \mathrm{~m}$ depth in Mar Virado Bay seems to follow the same pattern observed in Ubatuba Bay. This site has a high proportion of silt and clay and a higher percentage of organic matter compared to transects at the same depth in the other bays. The confluence of tropical waters from the Brazil Current with subantarctic waters from the Falklands Current occurs between latitudes $30^{\circ}$ and $46^{\circ} \mathrm{S}$. This subtropical convergence in the southwestern Atlantic (Pires 1992) forms a water mass called the South Atlantic Central Water (SACW). During certain times of the year, this water mass, depending on the intensity and influence of currents and winds (Castro-Filho et al. 1987), can influence coastal regions, causing horizontal and vertical changes, as well as seasonal mixing of tropical and subantarctic waters (Odebrecht and Castello 2001). Changes in bottom and surface temperatures and salinity are related to the hydrodynamics of the water masses in the Ubatuba region (see Pires 1992). These changes can lead to a higher abundance of breeding females in this location, since large-sized larvae can float more easily in high-salinity waters and are more easily dispersed by ocean currents (Pita et al. 1985, Mantelatto 2000). Johnson and Perry (1999) noted that seasonal circulation patterns driven by average wind stress allowed blue-crab larvae to be dispersed offshore and return to nearshore areas during the appropriate period in their development for settlement as megalopae.
Table 4. Demographic group scores, biplot scores, and correlations (interset and intraset) for the environmental variables with the ordination axes. Biplot scores are used to plot the vectors in the ordination diagram.

Tabla 4. Puntuaciones de los grupos demográficos, del diagrama de dispersión biespacial y las correlaciones (intergrupo e intragrupo) para las variables ambientales y los ejes de ordenación. Las puntuaciones de dispersión biespacial se usan para graficar los vectores en el diagrama de ordenación.

\begin{tabular}{|c|c|c|}
\hline & Axis 1 & Axis 2 \\
\hline \multicolumn{3}{|c|}{ Scores of demographic groups } \\
\hline Adult females & -0.87 & 1.42 \\
\hline Breeding females & -2.03 & -2.28 \\
\hline Adult males & 0.30 & -0.34 \\
\hline Immature individuals & 1.07 & -0.18 \\
\hline \multicolumn{3}{|c|}{ Scores of environmental variables } \\
\hline \multicolumn{3}{|l|}{ Biplot scores } \\
\hline Bottom temperature & 0.042 & -0.042 \\
\hline Salinity & -0.057 & 0.012 \\
\hline Organic matter & 0.063 & 0.004 \\
\hline Mean grain size (phi) & 0.132 & 0.005 \\
\hline \multicolumn{3}{|l|}{ Intraset correlation } \\
\hline Bottom temperature & 0.31 & -0.833 \\
\hline Salinity & -0.419 & 0.239 \\
\hline Organic matter & 0.467 & 0.08 \\
\hline Mean grain size (phi) & 0.97 & 0.105 \\
\hline \multicolumn{3}{|l|}{ Interset correlation } \\
\hline Bottom temperature & 0.154 & -0.245 \\
\hline Salinity & -0.209 & 0.07 \\
\hline Organic matter & 0.233 & 0.024 \\
\hline Mean grain size (phi) & 0.484 & 0.031 \\
\hline
\end{tabular}

dinámico y de alta energía (Pires 1992). Esto podría explicar la gran abundancia de cangrejos inmaduros en la bahía de Ubatuba, donde la alta concentración de materia orgánica y la cantidad de limo y arcilla permitieron una mayor disponibilidad de alimento para estos individuos, especialmente en la zona protegida y cerca de las costas rocosas (Mascaró et al. 2007). Santos et al. (1995) observaron una mayor abundancia de individuos inmaduros de Portunus spinimanus en agua somera (4 $\mathrm{m}$ de profundidad) en la bahía de Fortaleza (cerca de nuestra zona de estudio), donde se capturaron cangrejos de tan solo $10 \mathrm{~mm}$ AC. Las mayores cantidades de individuos inmaduros a menor profundidad en zonas protegidas podrían estar relacionadas con la disponibilidad de refugio y alimento, además de una alta productividad, resultando en altas tasas de crecimiento (Hines et al. 1987). En este estudio, se registró una abundancia de cangrejos inmaduros en el transecto de Ubatuba expuesto al oleaje, lo que se puede atribuir a la presencia de varias rocas grandes cerca del transecto. Los 
Adult males seem to display environmental preferences similar to those of immature individuals, possibly as a reproductive strategy to increase mating success by inhabiting areas where females undergo pubertal molt (changing from juveniles to adults). Santos (2000) suggested that when environmental conditions influence the reproduction of a population, the combination of prevailing environmental factors (physical, chemical, and biotic) should be considered, in addition to interactions such as competition, predation, and social structure. Callinectes ornatus occurs mainly in more saline, transparent, and deeper waters, and on fine- and medium-sand bottoms, that is, areas with greater marine influence (Carvalho and Couto 2011). The portunids Callinectes similis and $C$. sapidus migrate to more saline and stable waters to spawn and incubate their eggs (Aguilar et al. 2005). Migration of C. sapidus females to high-salinity waters tends to improve their reproductive success, because eggs and larvae will be subject to less fluctuation of physical and chemical parameters and also to lower predation pressure (Hines et al. 1987). Juvenile and adult males of C. danae may concentrate in low-salinity areas in bays, whereas females prefer more saline waters (Sforza et al. 2010). This partial sexual segregation is associated with the reproductive cycle of swimming crabs, and was mentioned for the first time by Norse (1977) in his study on the zoogeographical distributions of members of the genus Callinectes. Subsequently, other researchers also cited this differential occupation of habitat, mainly during breeding periods, such as Pita et al. (1985) and Hines et al. (1987) for C. danae and C. sapidus, respectively.

This differential habitat occupation by crabs might change according to temporal variations, given that, as previously reported, the different demographic groups prefer certain environmental conditions. Bottom and surface temperatures, as well as salinity, are the factors that oscillate the most during the year, due to the influence of SACW. These factors were significantly correlated, particularly with adult females, breeding females, and immature individuals, which are most abundant during months with higher temperatures and salinity (see Johnson and Perry 1999). These months are more favorable for reproduction, and during this period females migrate to deeper regions that are more suitable for larval hatching, while larvae migrate to shallower habitats to develop.

Not only immature individuals but also the smallest adult individuals of C. ornatus were found in protected areas. This is probably related to the presence of large amounts of organic detritus and the high organic-matter content in the sediment (see Mascaró et al. 2007). As suggested by Heck et al. (2001) for C. sapidus, juveniles may be more abundant in sheltered areas because of the reduced risk of predation and less competition for mates from smaller adult males. However, the smaller males may not succeed in fertilizing females, as the females may already have their seminal individuos inmaduros de algunas especies de protúnidos preferentemente usan hábitats salobres para mudar, posiblemente por las ventajas osmóticas y las menores tasas de depredación en estos sitios (Hines et al. 1987). En la bahía de Ubatuba se encontró el mayor número de individuos inmaduros de Persephona mediterranea, lo que sugiere que esta bahía proporciona condiciones favorables para éstos (Bertini et al. (2001). Además, se ha sugerido que existen diferences ontogenéticas en las estrategias de alimentación de Callinectes rathbunae, lo que también podría favorecer la presencia de cangrejos inmaduros cerca de la costa (Mascaró et al. 2007). En un estudio sobre la distribución batimétrica de cangrejos, Bertini y Fransozo (2004) observaron que la población de $C$. ornatus predomina entre 2 y $5 \mathrm{~m}$ de profundidad, donde hay una baja diversidad de especies. Esta distribución restringida a profundidades menores puede explicarse por la abundancia de individuos inmaduros, ya que cangrejos pequeños sobreviven mejor donde hay un menor número de depredadores y mejor protección (Buchanan y Stoner 1988).

La mayor abundancia de hembras ovígeras en el transecto establecido a $20 \mathrm{~m}$ en la bahía de Mar Virado parece seguir el mismo patrón que el observado en la bahía de Ubatuba. Este sitio tiene una proporción alta de limo y arcilla y un mayor porcentaje de materia orgánica en comparación con los transectos a $20 \mathrm{~m}$ de las otras bahías. Las aguas tropicales de la corriente de Brasil confluyen con las aguas subantárticas de la corriente de las Malvinas entre $30^{\circ}$ y $46^{\circ} \mathrm{S}$ de latitud. Esta convergencia subtropical en el Atlántico sudoccidental (Pires 1992) forma una masa de agua denominada Agua Central del Atlántico Sur (ACAS). En ciertas épocas del año, esta masa de agua, dependiendo de la intensidad e influencia de las corrientes y vientos (Castro-Filho et al. 1987), puede influenciar las regiones costeras, causando cambios horizontales y verticales, así como una mezcla temporal de las aguas tropicales y subantárticas (Odebrecht y Castello 2001). Los cambios de la temperatura del fondo y superficial y de salinidad están relacionados con la hidrodinámica de las masas de agua en la región de Ubatuba (ver Pires 1992). Estos cambios pueden resultar en una mayor abundancia de hembras ovígeras en este sitio, ya que las larvas grandes pueden flotar más fácilmente en aguas con salinidades altas y son dispersadas con mayor facilidad por las corrientes oceánicas (Pita et al. 1985, Mantelatto 2000). Johnson y Perry (1999) observaron que los patrones de circulación estacional generados por la fuerza del viento permiten que las larvas de cangrejo azul se dispersen en mar abierto y regresen a zonas costeras durante el periodo apropiado de su desarrollo para el asentamiento de megalopas.

Los machos adultos muestran preferencias ambientales similares a las de individuos inmaduros, posiblemente como una estrategia reproductiva para incrementar el éxito de apareamiento, ocupando zonas donde las hembras experimentan la muda a la pubertad (cambian de hembras jóvenes a adultas). Santos (2000) sugirió que cuando las condiciones ambientales influyen en la reproducción de una población, se 
receptacles partly or completely filled with the spermatophore of another male (see Johnson 1980).

The normality of the size-class distribution in the $C$. ornatus population analyzed here indicates an equilibrium between birth and mortality rates, and also a balance between immigration and emigration rates, that is, a stable population structure (Negreiros-Fransozo et al. 1999). Brachyuran populations in tropical and subtropical regions generally show these features, as reported by Santos et al. (1995) for Portunus spinimanus in Fortaleza Bay, by Chacur and Negreiros-Fransozo (2001) for C. danae in Ubatuba Bay, and by Cobo (2005) for Mithraculus forceps at Couves Island (Ubatumirim). Males were distributed in all size classes, whereas females were concentrated in the intermediate classes. A similar pattern was found by Ripoli et al. (2007) for Portunus spinimanus in the state of Espírito Santo, southeastern Brazil. Differential growth between sexes, also shown by C. ornatus, may explain the higher abundance of females in the intermediate size classes (Santos et al. 1995), because in certain life stages females expend more energy on reproduction than on growth. Males were on average larger than females, which is the usual case for portunid crabs (Branco and Masunari 1992). This attribute is an important adaptation for mating, as larger males can better manipulate females and a larger size maximizes their potential for combat with other males (Mantelatto and Fransozo 1999).

This study revealed that the different demographic groups occupy the habitat in different ways, according to local features. Despite being part of the shrimp fishery bycatch, C. ornatus is the most common portunid in the region. In general, the region offers a range of different conditions that favor breeding and consequently the maintenance and growth of the local population of $C$. ornatus.

\section{ACKNOWLEDGMENTS}

The authors thank the Fundação de Amparo à Pesquisa do Estado de São Paulo (FAPESP) for providing financial support (\#97/12106-8; \#97/12108-6; \#97/12107-0). They are also greatful to Maria Lucia Negreiros-Fransozo for her constructive comments on early drafts of the manuscript, to NEBECC co-workers for their help during the fieldwork, and to Janet Reid for her valuable help with the English language. All sampling in this study was conducted in compliance with applicable Brazilian state and federal laws.

\section{REFERENCES}

Aguilar R, Hines AH, Wolcott TG, Wolcott DL, Kramer MA, Lipcius RN. 2005. The timing and route of movement and migration of post-copulatory female blue crabs, Callinectes sapidus Rathbun, from the upper Chesapeake Bay. J. Exp. Mar. Biol. Ecol. 319: 117-128. http://dx.doi.org/10.1016/j.jembe.2004.08.030

Bertini G, Fransozo A, Costa RC. 2001. Ecological distribution of three species of Persephona (Brachyura, Leucosiidae) in the Ubatuba region, São Paulo, Brazil. Nauplius 9: 31-41. deberían considerar tanto la combinación de factores ambientales prevalecientes (físicos, químicos y bióticos), como las interacciones bióticas como la competencia y depredación y la estructura social. Callinectes ornatus habita principalmente en aguas más profundas, salinas y transparentes, y en fondos de arena fina a media, esto es, en zonas con mayor influencia marina (Carvalho y Couto 2011). Los cangrejos portúnidos Callinectes similis y $C$. sapidus migran hacia aguas más estables y salinas para desovar e incubar sus huevos (Aguilar et al. 2005). La migración de hembras de C. sapidus hacia aguas de alta salinidad tiende a mejorar su éxito reproductivo, ya que los huevos y las larvas están sujetas a una menor fluctuación de los parámetros físicos y químicos, así como a una menor presión de depredación (Hines et al. 1987). Los machos jóvenes y adultos de $C$. danae pueden concentrarse en zonas de las bahías con baja salinidad, mientras que las hembras prefieren aguas de mayor salinidad (Sforza et al. 2010). Esta segregación sexual parcial se asocia con el ciclo reproductivo de cangrejos nadadores, y fue mencionado por primera vez por Norse (1977) en su estudio de las distribuciones zoogeográficas de los miembros del género Callinectes. Posteriormente, otros investigadores han citado esta ocupación diferencial de hábitats, principalmente durante los periodos reproductivos, tales como Pita et al. (1985) y Hines et al. (1987) para C. danae y C. sapidus, respectivamente.

Esta ocupación diferencial de hábitats de los cangrejos puede cambiar con variaciones temporales, dado que, como ya se ha mencionado, los diferentes grupos demográficos prefieren ciertas condiciones ambientales. La temperatura del fondo y superficial así como la salinidad son los factores que más oscilan durante el año debido a la influencia de ACAS. Estos factores se correlacionaron significativamente, especialmente con las hembras adultas y ovígeras y los individuos inmaduros, los cuales son más abundantes durante los meses cuando las temperaturas y la salinidad son mayores (ver Johnson y Perry 1999). Estos meses son más favorables para la reproducción, y durante este periodo las hembras migran hacia zonas más profundas para la eclosión larval, mientras que las larvas migran hacia hábitats más someros para su desarrollo.

No sólo los individuos inmaduros sino también los adultos más pequeños de $C$. ornatus se encontraron en áreas protegidas. Esto se puede atribuir a la presencia de grandes cantidades de detrito orgánico y al alto contenido de materia orgánica en el sedimento (ver Mascaró et al. 2007). Heck et al. (2001) sugirieron que individuos jóvenes de C. sapidus son más abundantes en zonas protegidas porque existe un menor riesgo de depredación y menos competencia para conseguir pareja con machos adultos de menor tamaño. Aún así, es posible que los machos más pequeños no logren fertilizar a las hembras, ya que los receptáculos seminales de las hembras podrían estar parcial o completamente llenos con los espermatóforos de otro macho (ver Johnson 1980). 
Bertini G, Fransozo A. 2004. Bathymetric distribution of brachyuran crab (Crustacea, Decapoda) communities on coastal soft bottoms off southeastern Brazil. Mar. Ecol. Prog. Ser. 279: 193-200.

Branco JO, Masunari S. 1992. Crescimento de Callinectes danae Smith (Decapoda, Portunidae) da Lagoa da Conceição, Florianópolis, Santa Catarina, Brasil. Rev. Bras. Zool. 9: 53-66.

Buchanan BA, Stoner AW. 1988. Distributional patterns of blue crabs (Callinectes sp.) in a tropical estuarine lagoon. Estuaries 11: 231-239.

Carvalho FL, Couto ECG. 2011. Environmental variables influencing the Callinectes (Crustacea: Brachyura: Portunidae) species distribution in a tropical estuary-Cachoeira River (Bahia, Brazil). J. Mar. Biol. Assoc. UK 91: 793-800. http://dx.doi.org/10.1017/S0025315410001700

Castro-Filho BM, Miranda LB, Miyao SY. 1987. Condições hidrográficas na Plataforma Continental ao largo de Ubatuba: Variações sazonais e em média escala. Bol. Inst. Oceanogr. 35: 135-151.

Chacur MM, Negreiros-Fransozo ML. 2001. Spatial and seasonal distributions of Callinectes danae (Decapoda, Portunidae) in Ubatuba Bay, São Paulo, Brazil. J. Crust. Biol. 21: 414-425. http://dx.doi.org/10.1651/02780372(2001)021[0414:SASDOC]2.0.CO;2

Cobo VJ. 2005. Population biology of the spider crab, Mithraculus forceps (A. Milne-Edwards 1875) (Majidae, Mithracinae) on the southeastern Brazilian coast. Crustaceana 78: 1079-1087.

Guillory V, Perry H, Steele P, Wagner T, Keithly W, Pellegrin B, Petterson J, Floyd T, Buckson B, Hartman L, Holder E, Moss C. 2001. The Blue Crab Fishery of the Gulf of Mexico, United States: A Regional Management Plan. Gulf States Marine Fisheries Commission, Ocean Springs, MS, 301 pp.

Heck Jr KL, Coen LD, Morgan SG. 2001. Pre- and post-settlement factors as determinants of juvenile blue crab Callinectes sapidus abundance: Results from the north-central Gulf of Mexico. Mar. Ecol. Prog. Ser. 222: 163-176.

Hines AH, Lipicus RN, Haddon AM. 1987. Population dynamics and habitat partitioning by size, sex and molt stage of blue crabs Callinectes sapidus in a subestuary of central Chesapeake Bay. Mar. Ecol. Prog. Ser. 36: 55-64.

Johnson DR, Perry HM. 1999. Blue crab larval dispersion and retention in the Mississippi Bight. Bull. Mar. Sci. 65: 129-149.

Johnson PT. 1980. Histology of the blue crab Callinectes sapidus: A model for the Decapoda. Praeger Scientific Publishing, New York, $440 \mathrm{pp}$.

Keunecke KA, D'Incao F, Verani JR, Vianna M. 2012. Reproductive strategies of two sympatric swimming crabs Callinectes danae and Callinectes ornatus (Crustacea: Portunidae) in an estuarine system, south-eastern Brazil. J. Mar. Biol. Assoc. UK 92: 343-347.

http://dx.doi.org/10.1017/S0025315411000397

Mantelatto FLM. 2000. Allocation of the portunid crab Callinectes ornatus (Decapoda: Brachyura) in the Ubatuba Bay, northern coast of São Paulo State, Brazil. In: Klein JCVV, Schram FR (orgs.), Crustacean Issues, The Biodiversity Crisis and Crustacea. AA Balkema, Rotterdam. Vol. 12, 1st ed., pp. 431-443.

Mantelatto FLM, Fransozo A. 1999. Characterization of the physical and chemical parameters of Ubatuba Bay, Northern coast of São Paulo State, Brazil. Rev. Bras. Biol. 59: 23-31. http://dx.doi.org/10.1590/S0034-71081999000100004

Mascaró M, Castillo AM, Simoes N, Chiappa-Carrara X. 2007. Variations in the feeding habits of Callinectes rathbunae in Las Palmas lagoon (southern Gulf of Mexico) on three temporal scales. Crustaceana 80: 139-160.
La normalidad de la distribución de las clases de talla de la población de $C$. ornatus analizada aquí indica un equilibrio entre las tasas de nacimiento y mortalidad, así como un balance entre las tasas de inmigración y emigración, esto es, una estructura de población estable (Negreiros-Fransozo et al. 1999). Las poblaciones de braquiuros en regiones tropicales y subtropicales generalmente muestran estas características, como ha sido documentado por Santos et al. (1995) para Portunus spinimanus en la bahía de Fortaleza, por Chacur y Negreiros-Fransozo (2001) para C. danae en la bahía de Ubatuba y por Cobo (2005) para Mithraculus forceps en la isla de Couves (Ubatumirim). Se observaron machos en todas las clases de talla, mientras que las hembras se concentraron en las clases intermedias. Ripoli et al. (2007) encontraron un patrón similar para Portunus spinimanus en el estado de Espírito Santo, en el sureste de Brasil. Un crecimiento diferencial por sexos, como la demuestra C. ornatus, puede explicar la mayor abundancia de hembras en las clases de talla intermedias (Santos et al. 1995), ya que en algunas etapas de su vida las hembras gastan más energía en la reproducción que en el crecimiento. Los machos fueron en promedio más grandes que las hembras, lo cual es común para cangrejos portúnidos (Branco y Masunari 1992). Este atributo es una adaptación importante para el apareamiento, ya que los machos grandes pueden manipular mejor a las hembras y un mayor tamaño maximiza su potencial de combate con otros machos (Mantelatto y Fransozo 1999).

Este trabajo mostró que los diferentes grupos demográficos ocupan el hábitat de diferentes maneras, según las características locales. A pesar de formar parte de la fauna de acompañamiento de la pesca de camarón, C. ornatus es el cangrejo portúnido más común de la región. En general, la región ofrece diversas condiciones que favorecen la reproducción y consecuentemente la continuidad y el crecimiento de la población local de C. ornatus.

\section{Agradecimientos}

Este trabajo fue financiado por la Fundação de Amparo à Pesquisa do Estado de São Paulo (FAPESP, \#97/12106-8; \#97/12108-6; \#97/12107-0). Los autores agradecen a Maria Lucia Negreiros-Fransozo sus comentarios constructivos a una versión anterior del manuscrito, a trabajadores del NEBECC su ayuda en el campo y a Janet Reid su valiosa ayuda con el idioma inglés. Todo el trabajo de campo se realizó conforme a leyes federales y estatales brasileñas.

Traducido al español por Christine Harris. 
McCune B, Grace JB. 2002. Analysis of Ecological Communities. MJM Software Design, Gleneden Beach, Oregon, 300 pp.

Melo GAS. 1996. Manual de Identificação dos Brachyura (Caranguejos e Siris) do Litoral Brasileiro. São Paulo, Pleiade/ FAPESP, 604 pp.

Negreiros-Fransozo ML, Mantelatto FLM, Fransozo A. 1999. Population biology of Callinectes ornatus Ordway, 1863 (Decapoda, Portunidae) from Ubatuba (SP), Brazil. Sci. Mar. 63: 157-163. http://dx.doi.org/10.3989/scimar.1999.63n2157

Norse EA. 1977. Aspects of the zoogeographic distribution of Callinectes (Brachyura: Portunidae). Bull. Mar. Sci. 27: 440-447.

Odebrecht C, Castello JP. 2001. The convergence ecosystem in the southwest Atlantic. In: Seeliger U, Kjerfve B (eds.), Ecological Studies: Coastal Marine Ecosystems of Latin America. Vol. 144. Springer Verlag, Berlin, pp. 147-166.

Pires AMS. 1992. Structure and dynamics of benthic megafauna on the continental shelf offshore of Ubatuba, southeastern Brazil. Mar. Ecol. Prog. Ser. 86: 63-76.

Pita JB, Rodrigues ES, Graça-Lopes R, Coelho JAP. 1985. Observações bioecológicas sobre o siri Callinectes danae Smith, 1869 (Crustacea, Portunidae) no complexo baía-estuário de Santos, Estado de São Paulo, Brasil. Bol. Inst. Pesca 12: $35-43$.

Rakocinski CF, Lyczkowski-Shultz J, Richardson SL. 1996. Ichthyoplankton assemblage structure in Mississippi Sound as revealed by canonical correspondence analysis. Estuar. Coast. Shelf Sci. 43: 237-257. http://dx.doi.org/10.1006/ecss.1996.0067

Ripoli LV, Fernandes JM, Rosa DM, Araujo CCV. 2007. Dinâmica populacional de Portunus spinimanus Latreille 1819 (Crustacea, Portunidae) em um trecho litorâneo da Ilha do Frade, Vitória, ES. Bol. Inst. Pesca 33: 205-212.

Santos S. 2000. Influência dos fatores ambientais na abundância de Portunus spinimanus Latreille 1819 (Crustacea, Brachyura, Portunidae) na Região de Ubatuba (SP). Ciênc. Nat. 22: 129-144.

Santos S, Negreiros-Fransozo ML, Fransozo A. 1995. Estructura poblacional de Portunus spinimanus Latreille 1819 (Decapoda, Brachyura, Portunidae) en la Enseada de la Fortaleza, Ubatuba (SP), Brasil. Rev. Invest. Mar. 16: 37-42.

Sforza R, Nalesso RC, Joyeux JC. 2010. Distribution and population structure of Callinectes danae (Decapoda: Portunidae) in a tropical Brazilian estuary. J. Crust. Biol. 30: 597-606. http://dx.doi.org/10.1651/09-3223.1

Wentworth CK. 1922. A scale of grade and class terms for clastic sediments. J. Geol. 30: 377-392.

Wilson K, Hardy ICW. 2002. Statistical analysis of sex ratios: an introduction. In: Hardy ICW (ed.), Sex Ratios: Concepts and Research Methods. Cambridge, University Press, 1st ed., pp. 48-92.

Zar JH. 1996. Biostatistical Analysis. 3rd ed. Prentice-Hall, New Jersey, $918 \mathrm{pp}$.

Received February 2013, Received in revised form October 2013, accepted October 2013. 